\title{
A Database of Selected Transport Coefficients for Combustion Studies
}

\author{
Lawrence D. Cloutman
}

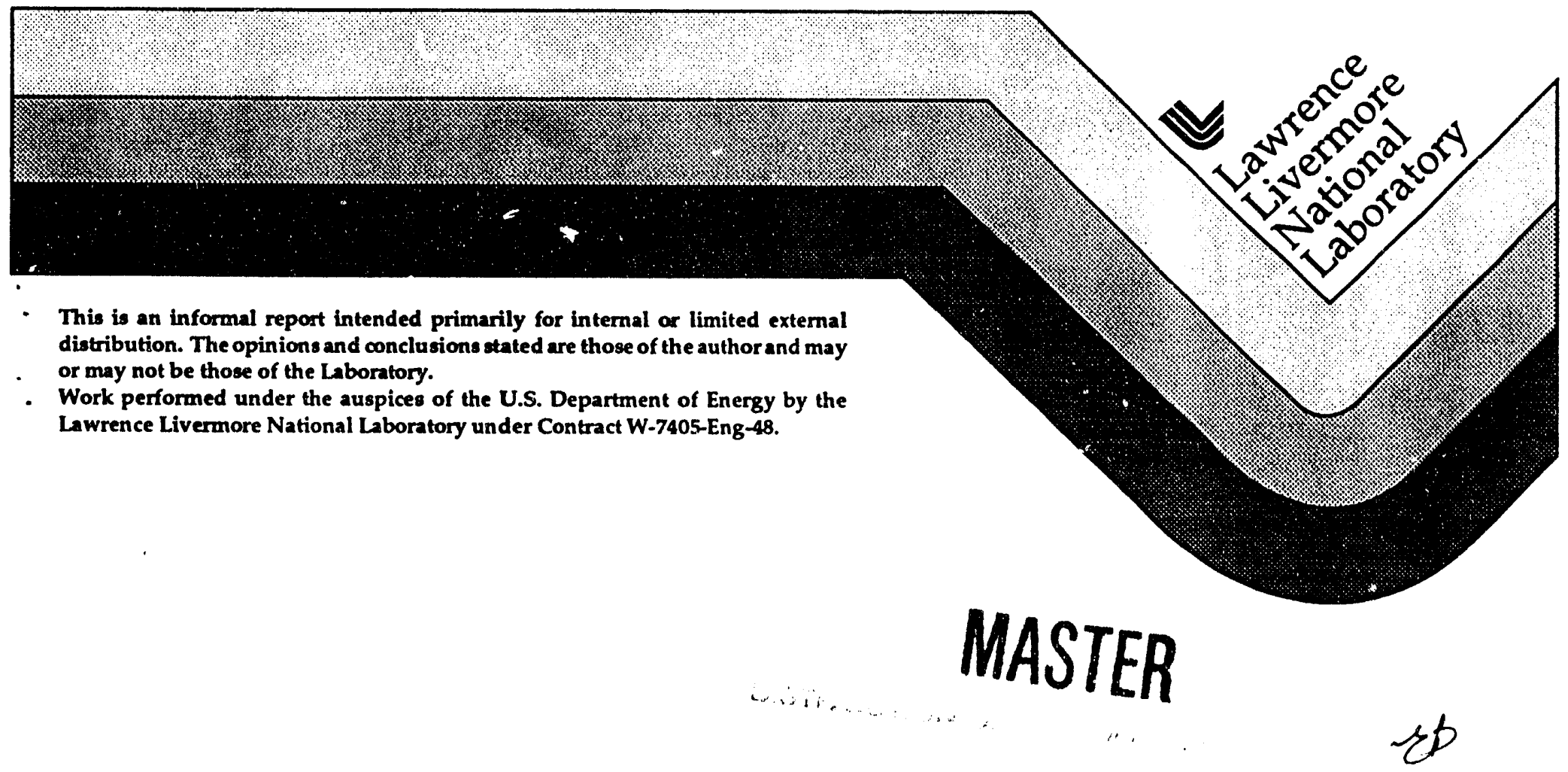




\section{DISCLAIMER}

This document was prepared as an accoount of work sponsored by an agency of the United States Government. Neither the United States Government nor the University of Califomia nor any of their employees, makes any warranty, express or implied, or assumes any legal liabillty or responsibility for the accuracy, completeness, or usefulness of any information, apparatus, product, or process disclosed, or represents that its use would not infringe privately own rights. Reference herein to any specific commercial products, process, or service by trade name, trademiark, manufacture, or olierwise, does not necessarily constitute or imply its endorsement, recommendation, or favoring by the United States Government or the University of California. The views and opinions of authors expressed herein do not necessarily state or reflect those of the United States Government or the University of California, and shall not be used for advertising or product endorsement purposes.

This report has been reproduced directly from the best available copy.

Available to DO': and DOE contractors from the Office of Scientific and Technical Information P.O. Box 62, Oak Ridge, IN 37831

Prices available from (615) 576-8401, FTS 626-8401

Avallable to the public from the National Technical information Service

U.S. Department of Commence 5285 Port Royal Rd. Springfield, VA 22161 


\title{
A DATABASE OF SELECTED TRANSPORT COEFFICIENTS FOR COMBUSTION ŚTUDIES
}

\author{
Lawrence D. Cloutman \\ Lawrence Livermore National Laboratory \\ Livermore, California 94550
}

\begin{abstract}
COYOTE and similar combustion programs based on the multicomponent Navier-Stokes equations require the mixture viscosity, thermal conductivity, and species transport coefficients as input. This report documents a significant improvement to the calculation of these molecular transport coefficients in COYOTE and provides a self-contained and easy-to-use source of such data in a format suitable for use by such programs. We present the data for various species in two forms. The first is a simple functional fit to the transport coefficients. The second is the use of tabulated Lennard-Jones parameters in simple theoretical expressions for the gas-phase transport coefficients. Tables are given for a number of chemical species.
\end{abstract}




\section{Introduction}

Viscosity, thermal conductivity, and species transport coefficients are an important part of any effort to make realistic numerical simulations of reactive flows, especially in the laminar regime. Unfortunately, the available data are scattered widely throughout the literature of chemistry, physics, and engineering in a wide variety of formats. This information must be collected into one place, assessed for accuracy, and put into the appropriate format before it can be used in any particular computational fluid dynamics (CFD) program. Due to the large number of species of potential interest in combustion studies (potentially a thousand or more for a single problem), creating and maintaining such a database can become a major task. The program system CHEMKIN [1-4] is an excellent example of a computerized database designed to address this need. It is difficult to overstate the importance of such a program system to users who are willing to live with the constraints and limitations inherent in using software developed by someone else. The obvious advantages are avoidance of the effort required to independently collect the data in a usable form and the ability to use the same gas physics as a wide community of researchers. However, there are also disadvantages. First, there is the effort of obtaining and porting the programs to the user's system and learning to use them. Second, there is the need to validate, maintain, and update the programs. This can be a major task, especially if local modifications have been made to the program system. Third, an interface with the user's hydrodynamics program must be created and maintained. Fourth, users are constrained by the physics and output formats provided unless they make local modifications.

This report presents a simpler solution to the problem of supplying transport coefficients to the COYOTE reactive-flow CFD program [5]. The first step is to select a representation of the transport coefficients for use in the CFD program. Two of the many possible choices are described in the following sections. These two choices are a simple parametric fitting function (a slightly generalized Sutherland equation) and the use of the Lennard-Jones parameters in simple analytical expressions for the transport coefficients. Sample data for both approaches are given in the tables in

this report. A text editor may be used to put these parameter values in to the COYOTE input file. 
In both cases, simple mixture rules are used to get viscosity and conductivity for a mixture with an arbitrary composition. These formulas are described in detail in the following sections. This is similar to the approach used in COYOTE with respect to the caloric equation of state. The JANAF tables [6-8] provide a homogeneous set of thermochemical data for a large collection of materials. The enthalpy tables and heats of formation for all species of interest are stored in a simple database program, GASMAK, which is run at the start of each problem to generate an input file containing the data on each species included in that problem. COYOTE then uses interpolation in the enthalpy table.

In Section II, we present the data for the coefficient of viscosity. Section III presents the thermal conductivities. Species diffusion coefficients are given in Section IV. Section V describes the input variables used by COYOTE's implementation of the transport coefficient data described in the previous sections. Section VI contains some concluding comments.

\section{The Coefficient of Viscosity}

Let $\mathcal{T}$ be the viscous stress tensor for a Newtonian fluid,

$$
\begin{gathered}
\mathcal{T}=-\mu\left[\nabla \vec{u}+(\nabla \vec{u})^{T}\right]-\mu_{1}(\nabla \cdot \vec{u}) \mathcal{U} \\
=-\mu\left[\nabla \vec{u}+(\nabla \vec{u})^{T}\right]+\left(\frac{2 \mu}{3}-\mu_{b}\right)(\nabla \cdot \vec{u}) \mathcal{U} .
\end{gathered}
$$

Here $\vec{u}$ is the velocity of the fluid mixture, $\mathcal{U}$ is the unit tensor, $\mu$ is the coefficient of viscosity, $\mu_{1}$ is the second coefficient of viscosity, and $\mu_{b}$ is the bulk viscosity. In almost all studies, the bulk viscosity is set to zero, which is correct, strictly speaking, only for perfect monatomic gases. What we require is a procedure for specifying the coefficient of viscosity for the mixture of species composing the fluid.

The first step in calculating the mixture viscosity is to evaluate the viscosity for each species. At the pressures and temperatures found in typical combustion systems, the species coefficients of viscosity are almost independent of density, so we assume they are functions only of temperature. 
Table 1.

Coefficient of Viscosity

\begin{tabular}{lllllll}
\hline \hline Species & $n_{\alpha}$ & $A_{\alpha}$ & $B_{\alpha}$ & $C_{\alpha}$ & $T$ Range & Ref. \\
\hline Air & 1.5 & $1.457 \times 10^{-5}$ & 110. & 0.0 & Unknown & - \\
$\mathrm{Air}$ & 1.51 & $1.387 \times 10^{-5}$ & 105.1 & 0.0 & $79-1407$ & 12 \\
$\mathrm{He}$ & 1.695 & $3.614 \times 10^{-6}$ & -9.549 & 0.0 & $81-1090$ & 12 \\
$\mathrm{~N}_{2}$ & 1.52 & $1.186 \times 10^{-5}$ & 86.54 & 0.0 & $251-1098$ & 12 \\
$\mathrm{O}_{2}$ & 1.46 & $2.294 \times 10^{-5}$ & 164.4 & 0.0 & $273-1102$ & 12 \\
$\mathrm{O}_{2}$ & 1.5 & $1.16 \times 10^{-5}$ & 0.0 & 0.0 & - & 13 \\
$\mathrm{C}_{3} \mathrm{H}_{8}$ & 1.72 & $1.802 \times 10^{-6}$ & 101.0 & 0.0 & $290-472$ & 12 \\
$\mathrm{SF}_{6}$ & 1.7 & $3.526 \times 10^{-6}$ & 73.1 & 0.0 & $223-573$ & 17 \\
$\mathrm{H}_{2}$ & 1.67 & $1.963 \times 10^{-6}$ & 2.187 & 0.0 & $89-1098$ & 12 \\
$\mathrm{H}$ & 1.5 & $3.95 \times 10^{-6}$ & 0.0 & 0.0 & - & 13 \\
0 & 1.5 & $1.15 \times 10^{-5}$ & 0.0 & 0.0 & - & 13 \\
$\mathrm{OH}$ & 1.5 & $1.10 \times 10^{-5}$ & 0.0 & 0.0 & - & 13 \\
$\mathrm{H}_{2} \mathrm{O}$ & 1.5 & $1.60 \times 10^{-5}$ & 0.0 & 0.0 & - & 13 \\
$\mathrm{HO}_{2}$ & 1.5 & $1.16 \times 10^{-5}$ & 0.0 & 0.0 & - & 13 \\
$\mathrm{H}_{2} \mathrm{O}_{2}$ & 1.5 & $1.16 \times 10^{-5}$ & 0.0 & 0.0 & - & 13 \\
\hline
\end{tabular}

We allow two ways of specifying the coefficient of viscosity. The first is a simple three-parameter analytic function that is suitable for fitting tabulated viscosity data. The second form is based on the Lennard-Jones parameters for each gas species.

The first approach is based on a fitting function that takes advantage of the fact that simple kinetic theory predicts that the viscosity of a gas is proportional to $T^{1 / 2}$, where $T$ is the absolute temperature. We adopt a slightly more complex parameterization, a generalized Sutherland formula as our fitting function:

$$
\mu_{\alpha}=\frac{A_{\alpha} T^{n_{\alpha}}}{B_{\alpha}+T}+C_{\alpha}
$$

where $\alpha$ denotes the particular species, and $A_{\alpha}, B_{\alpha}, C_{\alpha}$, and $n_{\alpha}$ are constants. Values of the constants are giver in Table 1 for a selection of species. The units are all $\mathrm{cgs}$, that is poise $(\mathrm{g} / \mathrm{cm}-\mathrm{s})$ for the viscosity and kelvins for $T$. To convert to SI units, multiply the cgs viscosity by 0.1 . The 
constants in Table 1 were evaluated by setting $\widehat{C}_{\alpha}=0.0$ and fitting the viscosity data in the references at three temperatures (usually at the extremes and middle of the temperature range provided). The constant $C_{\alpha}$ is provided mostly as a convenience in setting the viscosity to a constant value in the CFD code for certain numerical experiments, although it could also have been used to fit a fourth data point. A least squares fit also could have been used.

The accuracy of the fits varies somewhat. Most of the fits in Table 1 have errors of at most a few percent in the temperature range given in the table, and they are often better than one percent over wide temperature ranges. Extrapolation beyond the given temperature range is always risky, although much less so on the high temperature end. Strong non-ideal gas effects can occur at low temperature that are not accounted for in the fits. For example, the He fit is approximately a factor of two in error around $20 \mathrm{~K}$, and it gets worse rapidly as the singularity at $9.549 \mathrm{~K}$ is approached.

The second approach uses the Lennard-Jones parameters to estimate the viscosity (in cgs units),

$$
\mu_{\alpha}=\frac{5}{16}\left(\frac{m_{\mathrm{H}} k_{\mathrm{B}}}{\pi}\right)^{1 / 2} \frac{\left(M_{\alpha} T\right)^{1 / 2}}{\sigma_{\alpha}^{2} \Omega_{\nu}}=2.6693 \times 10^{-5} \frac{\left(M_{\alpha} T\right)^{1 / 2}}{\sigma_{\alpha}^{2} \Omega_{\nu}}
$$

where $M_{\alpha}$ is the molecular weight, $m_{\mathrm{H}}$ is the mass of one atomic mass unit in grams, $k_{\mathrm{B}}$ is the Boltzmann constant, $\sigma_{\alpha}$ is the collision diameter in $\AA$, and $\Omega_{\nu}$ is the collision integral approximated by

$$
\Omega_{\nu}=1.147\left(T / T_{e \alpha}\right)^{-0.145}+\left(T / T_{e \alpha}+0.5\right)^{-2}
$$

where $T / T_{e \alpha}=T k_{\mathrm{B}} / \epsilon_{\alpha}$ is the reduced temperature and $\epsilon_{\alpha}$ is the Lennard-Jones potential well depth [9, 10]. Kee, et al. [2] recommend interpolation in Table V of Monchick and Mason [11], which includes dependence of the collision integral on the reduced dipole r oment of the molecule, $\delta$, as well as dependence on $T_{e \alpha}$. Equation (4) is accurate to a few percent for Monchick and Mason's $\delta<0.5$ at low temperatures, and it becomes valid for larger values of $\delta$ at higher temperatures.

Once the species viscosities have been calculated, they must be combined to provide the viscosity of the fluid mixture. We adopt Wilke' law [12] as modified by Bird et al. [13]. For $N$ species,

$$
\mu=\sum_{\alpha=1}^{N} \frac{X_{\alpha} \mu_{\alpha}}{\sum_{\beta=1}^{N} X_{\beta} \Phi_{\alpha \beta}}
$$


where $X_{\alpha}$ is the mole fraction of species $\alpha$ and where

$$
\Phi_{\alpha \beta}=8^{-1 / 2}\left(1+\frac{M_{\alpha}}{M_{\beta}}\right)^{-1 / 2}\left[1+\left(\frac{\mu_{\alpha}}{\mu_{\beta}}\right)^{1 / 2}\left(\frac{M_{\beta}}{M_{\alpha}}\right)^{1 / 4}\right]^{2} .
$$

\section{Thermal Conductivities}

The heat flux is a complicated function, and COYOTE uses

$$
\vec{q}=-K \nabla T+\sum_{\alpha} h_{\alpha}(T) \vec{J}_{\alpha}-\sum_{\alpha} \frac{R T}{M_{\alpha} X_{\alpha}} D_{\alpha}^{T}\left[\nabla X_{\alpha}+\left(X_{\alpha}-Y_{\alpha}\right) \frac{\nabla P}{P}\right]
$$

where $K$ is the mixture thermal conductivity, $h_{\alpha}$ is the specific enthalpy of species $\alpha, \vec{J}_{\alpha}$ is the species mass flux, and $D_{\alpha}^{T}$ is the thermal diffusion coefficient [2]. Calculation of the species mass fluxes and thermal diffusion coefficients will be considered in the next section.

In the original COYOTE program, $K$ was calculated from the mixture viscosity $\mu$ and a constant mixture Prandtl number. This capability has been expanded to allow calculation of the conductivity based on the local composition and temperature, just. as was done for the viscosity. One option is to fit species conductivity data using the same functional form as for the viscosity (that is, Eq. (2)). Results of a few fits are given in Table 2.

The second option is based on the Lennard-Jones parameters. Following the procedure of Hayashi and Hishida [9], we calculate the conductivity from the viscosity using the Eucken correction, which is discussed also by Ferziger and Kaper [14]:

$$
K_{\alpha}=0.25\left(9 \gamma_{\alpha}-5\right) \mu_{\alpha} C_{\mathrm{v} \alpha}
$$

where $C_{\mathrm{v} \alpha}$ is the specific heat at constant volume and $\gamma_{i}$ is the ratio of specific heats. Kee, et al. [2] describe a more complex approximation that we have not implemented. Their model accounts more accurately for the internal quantum states of the molecules.

There are two possible mixture rules for the conductivity. The first is to use Eq. (5) with $\mu_{\alpha}$ replaced by $K_{\alpha}$, but with the same values of $\Phi_{\alpha \beta}$ as used for the mixture viscosity [13]. The second 
Table 2.

Species Th.rmal Conductivities

\begin{tabular}{lllllll}
\hline \hline Species & $n_{\alpha}$ & $A_{\alpha}$ & $B_{\alpha}$ & $C_{\alpha}$ & $T$ Range & Ref. \\
\hline $\mathrm{Air}$ & 1.5 & $2.255 \times 10^{2}$ & 150.0 & 0.0 & $233-322$ & 16 \\
$\mathrm{He}$ & 1.63 & $4.167 \times 10^{2}$ & 2.635 & 0.0 & $33-322$ & 16 \\
$\mathrm{~N}_{2}$ & 1.51 & $1.935 \times 10^{2}$ & 110.4 & 0.0 & $88-322$ & 16 \\
$\mathrm{O}_{2}$ & 1.86 & $2.126 \times 10^{1}$ & 22.26 & 0.0 & $88-366$ & 16 \\
$\mathrm{C}_{3} \mathrm{H}_{8}$ & 2.69 & $1.167 \times 10^{-1}$ & 2.409 & 0.0 & $233-366$ & 16 \\
$\mathrm{SF}_{6}$ & 1.8 & $2.296 \times 10^{1}$ & 140.5 & 0.0 & $300-4000$ & 17 \\
$\mathrm{H}_{2}$ & 1.5 & $1.522 \times 10^{3}$ & 124.4 & 0.0 & $88-322$ & 16 \\
$\mathrm{H}$ & 1.5 & $1.173 \times 10^{3}$ & 0.0 & 0.0 & - & 18 \\
$\mathrm{O}$ & 1.5 & $2.134 \times 10^{2}$ & 0.0 & 0.0 & - & 18 \\
$\mathrm{OH}$ & 1.5 & $2.69 \times 10^{2}$ & 0.0 & 0.0 & - & 18 \\
$\mathrm{H}_{2} \mathrm{O}$ & 1.5 & $4.596 \times 10^{2}$ & 1036.2 & 0.0 & $255-367$ & 16 \\
\hline
\end{tabular}

rule is due to Mathur et al. [15] and is recommended by Kee, et al. [2]:

$$
K=\frac{1}{2}\left[\sum_{\alpha=1}^{N} X_{\alpha} K_{\alpha}+\left(\sum_{\alpha=1}^{N} X_{\alpha} / K_{\alpha}\right)^{-1}\right] .
$$

We presently use the latter rule in COYOTE.

\section{Species Diffusion Coefficients}

Calculation of the exact diffusional mass fluxes is an extremely complicated task (for example, [13], [19], and [20]). Many combustion studies simply use Fick's law,

$$
\vec{J}_{\alpha}=-\rho D_{\alpha} \nabla\left(\rho_{\alpha} / \rho\right)
$$

where $\rho$ is the total density, and $D_{\alpha}$ is the species diffusivity. The original version of COYOTE used this approximation with the same value of $D_{\alpha}$ for all species, and this value was given as the kinematic viscosity of the mixture divided by a constant mixture Schmidt number. This simple model has the advantages that it is easy to program, computationally inexpensive, and the species mass 
fluxes properly add up to zero when summed over species. Experience suggests that it is adequate for turbulent flows (where molecular transport is overwhelmed by the turbulent eddy diffusion) and in some laminar cases using global chemical kinetics (where the detailed chemical composition is not being modeled all that accurately in any case). In cases where the flow is laminar or nearly so and a detailed chemical reaction network is included, a more accurate model is required.

Ramshaw [21] has developed an effective binary diffusion approximation that we have adopted. He begins with the assumption that multicomponent diffusion in gases is governed by the StefanMaxwell equations

$$
\sum_{\beta}\left(X_{\alpha} X_{\beta} / D_{\alpha \beta}\right)\left(\vec{u}_{\beta}-\vec{u}_{\alpha}\right)=\vec{G}_{\alpha} \quad(\alpha=1, \ldots N),
$$

where $N$ is the number of species in the mixture, $\vec{u}_{\alpha}$ is the velocity of species $\alpha, X_{\alpha}$ is the mole fraction of species $\alpha, D_{\alpha \beta}$ is the binary diffusivity for the pair of species $(\alpha, \beta)$, and the driving forces $\vec{G}_{\alpha}$ are given by

$$
\vec{G}_{\alpha}=\nabla X_{\alpha}+\left(X_{\alpha}-Y_{\alpha}\right) \nabla \ln P+\chi_{\alpha} \nabla \ln T-\frac{1}{P}\left[\rho_{\alpha} \vec{F}_{\alpha}-Y_{\alpha} \sum_{\beta} \rho_{\beta} \vec{F}_{\beta}\right]
$$

Here $\rho_{\alpha}$ is the mass density of species $\alpha, \rho$ is the total density of the mixture, $Y_{\alpha}$ is the mass fraction of species $\alpha$, and $\vec{F}_{\alpha}$ is the body force per unit mass acting on species $\alpha$. The $\chi_{\alpha}$ are related to the species thermal diffusion coefficients. The diffusive mass fluxes are given by

$$
\vec{J}_{\alpha}=\rho_{\alpha}\left(\vec{u}_{\alpha}-\vec{u}\right)
$$

where $\vec{u}$ is the mass-weighted mixture velocity calculated by COYOTE,

$$
\rho \vec{u}=\sum_{\alpha} \rho_{\alpha} \vec{u}_{\alpha}
$$

The diffusional fluxes could be found by solving the coupled system (11) through (13) for each cell on each time step, a complex and expensive process when more than two species are present. Another approach is to decouple the fluxes from one another with what is called the "effective binary diffusion approximation," which exists in a variety of forms. In the absence of thermal, forced, and 
pressure diffusion, Eq. (10) is a commonly used approximation. However, this approximation has the serious disadvantage that the fluxes do not sum to zero unless all values of $D_{\alpha}$ are the same.

Ramshaw's contribution is the development of an effective binary diffusion approximation that simultaneously accounts for all four types of diffusion, and for which the fluxes correctly sum to zero. The resulting model is

$$
\vec{J}_{\alpha}=-c M_{\alpha} D_{\alpha} \vec{G}_{\alpha}+Y_{\alpha} c \sum_{\beta} M_{\beta} D_{\beta} \vec{G}_{\beta}
$$

where $M_{\alpha}$ is the molecular weight of species $\alpha$,

$$
c=\sum_{\alpha} \rho_{\alpha} / M_{\alpha}
$$

is t?

$$
D_{\alpha}=\left(1-X_{\alpha}\right)\left(\sum_{\beta \neq \alpha} X_{\beta} / D_{\alpha \beta}\right)^{-1}
$$

The interested reader is referred to Ramshaw [21] for details.

We follow Hayashi and Hishida [9] in evaluation of the binary diffusion coefficients:

$$
D_{\alpha \beta}=1.8829 \times 10^{3} \frac{\left[T^{3}\left(\frac{M_{\alpha}+M_{\beta}}{M_{\alpha} M_{\beta}}\right)\right]^{1 / 2}}{P \sigma_{\alpha \beta}^{2} \Omega_{\mathrm{D}}},
$$

where $P$ is the total pressure of the mixture. We also use

$$
\begin{gathered}
\sigma_{\alpha \beta}=0.5\left(\sigma_{\alpha}+\sigma_{\beta}\right) \\
\Omega_{\mathrm{D}}=\left(T / T_{e \alpha \beta}\right)^{-0.145}+\left(T / T_{e \alpha \beta}+0.5\right)^{-2}
\end{gathered}
$$

and

$$
T_{e \alpha \beta}=\left(T_{e \alpha} T_{e \beta}\right)^{1 / 2}
$$

COYOTE presently uses $\chi_{\alpha}=D_{\alpha}^{T}=0$. Not only are thermal diffusion effects small, there are practical difficulties associated with obtaining the required values of $\chi_{\alpha}$. There is a shortage of experimental data, and the traditional theory is so complex as to hinder its application by the 
nonspecialist. However, Ramshaw has developed an approximate simplified theory [22] thai will be incorporated into COYOTE. The first step is to estimate the collision cross section between molecules of types $\alpha$ and $\beta$ as

$$
\sigma^{\alpha \beta}=0.25 \pi\left(\sigma_{\alpha}+\sigma_{\beta}\right)^{2}
$$

We define

$$
\gamma_{\alpha \beta}=\frac{m_{\mathrm{H}}}{2 k_{\mathrm{B}} T} \frac{M_{\alpha} M_{\beta}}{M_{\alpha}+M_{\beta}} .
$$

With these two parameters we calculate the collision time

$$
\tau_{\alpha}=\frac{1}{2}\left[\sum_{\beta=1}^{N} \frac{n_{\beta} \sigma^{\alpha \beta}}{\left(\pi \gamma_{\alpha \beta}\right)^{1 / 2}}\right]^{-1},
$$

where $n_{\beta}$ is the number density of species $\beta$. We can now calculate the conventional multicomponent thermal diffusion coefficients

$$
D_{\alpha}^{T}=k_{\mathrm{B}} T\left(n_{\alpha} \tau_{\alpha}-Y_{\alpha} \sum_{\beta=1}^{N} n_{\beta} \tau_{\beta}\right)
$$

which are in turn used to calculate

$$
\chi_{\alpha}=\sum_{\beta=1}^{N} \frac{X_{\alpha} X_{\beta}}{D_{\alpha \beta}}\left(\frac{D_{\alpha}^{T}}{\rho_{\alpha}}-\frac{D_{\beta}^{T}}{\rho_{\beta}}\right)
$$

for use in the Stefan-Maxwell equations (12), which in turn are used with Eq. (15) to calculate the diffusion fluxes.

\section{COYOTE Input for Transport Coefficients}

The transport coefficient data is input into COYOTE as part of the NAMELIST input deck for each problem. The data are found in Tables 1, 2, and 3. Table 3 contains the Lennard-Jones parameters for the set of species used in Glarborg's skeletal mechanism for methane combustion and $\mathrm{NO}_{x}$ chemistry [23], which provides a selection of some of the most important species in combustion. Also included are several species that have been encountered on occasion, including the use of the data of Bzowski, et al. [24] to test the models proposed in this report. The format of the table 
follows the practice of CHEMKIN [2]. The Index variable is 0,1 , or 2 if the molecules are a single atorn, a linear molecule, or a nonlinear molecule. Parameters for additional species may be found in CHEMKIN and scattered throughout the literature. For example, Bird, Stewart, and Lightfoot [13] and Dean, Bozzelli, and Ritter [25] provide short lists of parameters. The latter paper also presents a theoretical model that estimates Lennard-Jones parameter values for other species.

The viscosity parameters are read into COYOTE in the NAMELIST blocks coydat and tranco. The following variables are set in coydat.

airmu $=0$, no Sutherland equation for air; $=1$, use the Sutherland equation for air $\left(A_{\alpha}=1.457 \times 10^{-5}, B_{\alpha}=110, n_{\alpha}=1.5\right.$, and $\left.C_{\alpha}=0.0\right)$ for the mixture viscosity.

xnumol $=$ constant molecular mixture kinematic viscosity.

jdrflg $=0$, use simple Fick's law with species diffusivities given by the mixture viscosity divided by the mixture Schmidt number scmol; $=1$, use the detailed binary diffusion coefficients and Ramshaw's algorithm for computing the species fluxes without thermal diffusion; $=2$, include thermal diffusion.

prmol $=$ constant molecular Prandtl number, used only with xnumol and the airmu=1.0 viscosity formula.

$s c m o l=$ constant molecular Schmidt number used when $j d r f l g=0$.

The following variables are set in NAMELIST block tranco. mixvis $=0$, use original transport coefficients (that is, use the parameters airmu and xnumol set in coydat); $=1$, use the species Sutherland formula fits for all species; $=2$, use the Lennard-Jones model for all species. visfa, visfb, visfc, visfn $=$ arrays containing the parameters $A_{\alpha}, B_{\alpha}, C_{\alpha}$, and $n_{\alpha}$ for mixvis $=1$; visfa and visfb contain the Lennard-Jones parameters $\sigma_{\alpha}$ (in $\AA$ ) and $T_{e \alpha}=\epsilon_{\alpha} / k_{\mathrm{B}}$ if mixvis $=2$. 
confa, confb, confc, confn = arrays containing the parameters analogous to visfa, visfb, visfc, visfn for the species conductivities. Used only for mixvis $=1$.

$j$ tdiff $=0$, no radiative transfer $;=1$, include gray $1 \mathrm{~T}$ radiation diffusion; $=2$, include gray $2 \mathrm{~T}$ diffusion; $=3$, include emission model radiative heat losses.

$j t c o, j t c o 2, j t h 2 o=$ species numbers for $\mathrm{CO}, \mathrm{CO}_{2}$, and $\mathrm{H}_{2} \mathrm{O}$ for use when $j$ tdiff $=3$.

\section{Concluding Comments}

This simplified, self-contained, and homogeneous data base has proved to be useful in combustion studies. The improved accuracy of the transport coefficients in COYOTE allows more detailed simulations of a wide variety of flows, especially in the laminar regime. For truly large numbers of species, a more automated program system such as CHEMKIN might be preferable, but for a typical COYOTE run with less than about 30 species, the present data base is much simpler to use. The option of direct usage of the Lennard-Jones parameters in COYOTE is preferred over the CHEMKIN approach of using the same theoretical expressions to calculate transport coefficients that are subsequently fit with polynomials, thereby introducing unnecessary truncation errors.

\section{Acknowledgements}

I thank J. D. Ramshaw for many useful discussions on various aspects of including molecular transport effects into computational fluid dynamics programs. I also thank Bob Kee, Lila Chase, and Bill Pitz for their help with CHEMKIN. This work was supported by the California Institute for Energy Efliciency and Southern California Gas Company. It was performed under the auspices of the U. S. Department of Energy by the Lawrence Livermore National Laboratory under contract number W-7405-ENG-48.

\section{REFERENCES}


1. R. J. Kee, J. A. Miller, and T. H. Jefferson, "CHEMKIN: A general-purpose, problem-independent, transportable, Fortran chemical kinetics code package," Sandia National Laboratories Report No. SAND80-8003, 1980.

2. R. J. Kee, G. Dixon-Lewis, J. Warnatz, M. E. Coltrin, and J. A. Miller, "A Fortran computer code package for the evaluation of gas-phase multicomponent transport properties," Sandia National Laboratories Report No. SAND86-8246, 1986; reprinted 1990.

3. R. J. Kee, F. M. Rupley, J. A. Miller, "The Chemkin thermodynamic data base," Sandia National Laboratories Report No. SAND87-8215, 1987.

4. R. J. Kee, F. M. Rupley, J. A. Miller, "Chemkin-II: A Fortran chemical kinetics package for the analysis of gas-phase chemical kinetics," Sandia National Laboratories Report No. SAND898009,1989 ; reprinted 1990.

5. L. D. Cloutman, "COYOTE: A computer program for $2 \mathrm{D}$ reactive flow simulations," Lawrence Livermore National Laboratory Report No. UCRL-ID-103611, 1990.

6. D. R. Stull and H. Prophet, "JANAF thernochemical tables, 2nd ed.," U. S. Department of Commerce/National Bureau of Standards Report No. NSRDS-NBS 37, 19.71.

7. M. W. Chase, J. L. Curnutt, A. T. Hu, H. Prophet, A. N. Syverud, and L. C. Walker, "JANAF thermochemical table, 1974 supplement," J. Phys. Chem. Ref. Data 3, 311 (1974).

8. M. W. Chase, Jr., C. A. Davies, J. R. Downey, Jr., D. J. Frurip, M. A. McDonald, and A. N. Syverud, "JANAF thermochemical tables, third edition, parts I and II," J. Phys. Chem. Ref. Data 14 Suppl. No. 1, (1985).

9. A. K. Hayashi and M. Hishida, "Numerical Study on Pulsed Jet Combustion," in Fourth International Symposium on Computational Fluid Dynamics, Vol. III, University of California at Davis, Sept. 9-12, 1991, pp. 19-24.

10. F. M. White, Viscous Fluid Flow (McGraw-Hill, New York, 1974).

11. L. Monchick and E. A. Mason, "Transport properties of polar gases," J. Chem. Phys. 35, 1676 (1961).

12. C. R. Wilke, "A Viscosity Equation for Gas Mixtures," J. Chem. Phys. 18, 517 (1950).

13. R. B. Bird, W. E. Stewart, and E. N. Lightfoot, Transport Phenomena (Wiley, New York, 1960).

14. J. H. Ferziger and H. G. Kaper, Mathematical Theory of Transport Processes in Gases (NorthHolland, Amsterdam, 1972).

15. S. Mathur, P. K. Tondon, and S. C. Saxena, "Thermal conductivity of binary, ternary, and quaternary mixtures of rare gases," Mol. Phys. 12, 569 (1967).

16. R. C. Weast, Ed., CRC Handbook of Chemistry and Physics, 60th Edition (CRC Press, Boca 
Raton, 1979).

17. W. Behrendt, N. Baumann, H.-J. Fachmann, P. Kuhn, and P. Merlet, Gmelin Handbuch der Anorganischen Chemie, 8th ed., edited by H. Bitterer (Springer-Verlag, Berlin, 1978), Suppl. Vol. 2, pp. 143-146.

18. E. Oran, T. Young, and J. Boris, "Application of time-dependent numerical methods to the description of reactive shocks," in Proceedings of the 17th Symposium (International) on Combustion (The Combustion Institute, Pittsburgh, 1979), pp. 43-54.

19. L. H. Aller and S. Chapman, "Diffusion in the sun," Ap. J. 132, 461 (1960).

20. S. Chapman and T. G. Cowling, 1 'he Mathematical Theory of Non-Uniform Gases (Cambridge University Press, London, 1952).

21. J. D. R,amshaw, "Self-consistent effective binary diffusion in multicomponent gas mixtures," J. Non-Equilib. Thermodyn. 15, 295 (1990).

22. J. D. Ramshaw, "Hydrodynamic theory of multicomponent diffusion and thermal diffusion in multitemperature gas mixtures," J. Non-Equilib. Thermodyn. (1993), in press.

23. P. Glarborg, N. I. Lilleheie, S. Byggstøyl, B. F. Magnussen, P. Kilpinen, and M. Hupa, "A reduced mechanism for nitrogen chemistry in methane combustion, " in Proceedings of the 24th Symposium (International) on Combustion (The Combustion Institute, Pittsburgh, 1993), in press.

24. J. Bzowski, J. Kestin, E. A. Mason, and F. J. Uribe, "Equilibrium and transport properties of gas mixtures at low density: eleven polyatomic gases and five noble gases," J. Phys. Chem. Ref. Data 19, 1179 (1990).

25. A. M. Dean, J. W. Bozelli, and E. R. Ritter, "CHEMACT: A computer code to estimate rate constants for chemically-activated reactions," Combust. Sci. and Tech. 80, 63 (1991). 
Table 3.

Selected Lennard-Jones Parameters

\begin{tabular}{|c|c|c|c|c|c|}
\hline Species & Index & $\epsilon / k_{\mathrm{B}}(\mathrm{K})$ & $\sigma(\AA)$ & $\mu$ (Debye) & $\alpha\left(\AA^{3}\right)$ \\
\hline $\mathrm{Ar}$ & 0 & 136.500 & 3.330 & 0.000 & 0.000 \\
\hline $\mathrm{C}$ & 0 & 71.400 & 3.298 & 0.000 & 0.000 \\
\hline $\mathrm{CH}$ & 1 & 80.000 & 2.750 & 0.000 & 0.000 \\
\hline $\mathrm{CH}_{2}$ & 1 & 144.000 & 3.800 & 0.000 & 0.000 \\
\hline $\mathrm{CH}_{3}$ & 1 & 144.000 & 3.800 & 0.000 & 0.000 \\
\hline $\mathrm{CH}_{4}$ & 2 & 141.400 & 3.746 & 0.000 & 2.600 \\
\hline $\mathrm{C}_{2} \mathrm{H}_{2}$ & 1 & 209.000 & 4.100 & 0.000 & 0.000 \\
\hline $\mathrm{CH}_{2} \mathrm{O}$ & 2 & 498.000 & 3.590 & 0.000 & 0.000 \\
\hline $\mathrm{CH}_{2} \mathrm{OH}$ & 2 & 417.000 & 3.690 & 1.700 & 0.000 \\
\hline $\mathrm{CN}$ & 1 & 75.000 & 3.856 & 0.000 & 0.000 \\
\hline $\mathrm{CO}$ & 1 & 98.100 & 3.650 & 0.000 & 1.950 \\
\hline $\mathrm{CO}_{2}$ & 1 & 244.000 & 3.763 & 0.000 & 2.650 \\
\hline H & 0 & 145.000 & 2.050 & 0.000 & 0.000 \\
\hline$\overline{\mathrm{H}}_{2}$ & 1 & 38.000 & 2.920 & 0.000 & 0.790 \\
\hline $\mathrm{HCN}$ & 1 & 569.000 & 3.630 & 0.000 & 0.000 \\
\hline $\mathrm{HCO}$ & 2 & 498.000 & 3.590 & 0.000 & 0.000 \\
\hline $\mathrm{H}_{2} \mathrm{O}$ & 2 & 809.100 & 2.641 & 1.850 & 0.000 \\
\hline $\mathrm{H}_{2} \mathrm{O}_{2}$ & 2 & 107.400 & 3.458 & 0.000 & 0.000 \\
\hline $\mathrm{HO}_{2}$ & 2 & 107.400 & 3.458 & 0.000 & 0.000 \\
\hline $\mathrm{N}$ & 0 & 71.400 & 3.298 & 0.000 & 0.000 \\
\hline $\mathrm{N}_{2}$ & 1 & 97.530 & 3.621 & 0.000 & 1.760 \\
\hline $\mathrm{NCO}$ & $\overline{1}$ & 232.400 & 3.828 & 0.000 & 0.000 \\
\hline $\mathrm{NH}$ & 1 & 80.000 & 2.650 & 0.000 & 0.000 \\
\hline $\mathrm{N}_{2} \mathrm{O}$ & 1 & 232.400 & 3.828 & 0.000 & 0.000 \\
\hline NO & 1 & 97.530 & 3.621 & 0.000 & 1.760 \\
\hline $\mathrm{NO}_{2}$ & 2 & 200.000 & 3.500 & 0.000 & 0.000 \\
\hline 0 & 0 & 80.000 & 2.750 & 0.000 & 0.000 \\
\hline $\mathrm{O}_{2}$ & 1 & 107.400 & 3.458 & 0.000 & 1.600 \\
\hline $\mathrm{OH}$ & 1 & 80.000 & 2.750 & 0.000 & 0.000 \\
\hline $\mathrm{C}_{2} \mathrm{H}_{4}$ & 2 & 280.800 & 3.971 & 0.000 & 0.000 \\
\hline $\mathrm{C}_{2} \mathrm{H}_{6}$ & 2 & 252.300 & 4.302 & 0.000 & 0.000 \\
\hline $\mathrm{C}_{3} \mathrm{H}_{8}$ & 2 & 266.800 & 4.982 & 0.000 & 0.000 \\
\hline $\mathrm{CF}_{4}$ & 2 & 156.500 & 4.579 & - & - \\
\hline $\mathrm{SF}_{6}$ & 2 & 207.700 & 5.252 & - & - \\
\hline $\mathrm{He}$ & 0 & 10.200 & 2.576 & 0.000 & 0.000 \\
\hline $\mathrm{Kr}$ & 0 & 176.0 & 3.679 & & \\
\hline $\mathrm{Ne}$ & 0 & 35.60 & 2.749 & & \\
\hline $\mathrm{Xe}$ & 0 & 229.8 & 3.937 & & \\
\hline
\end{tabular}


I
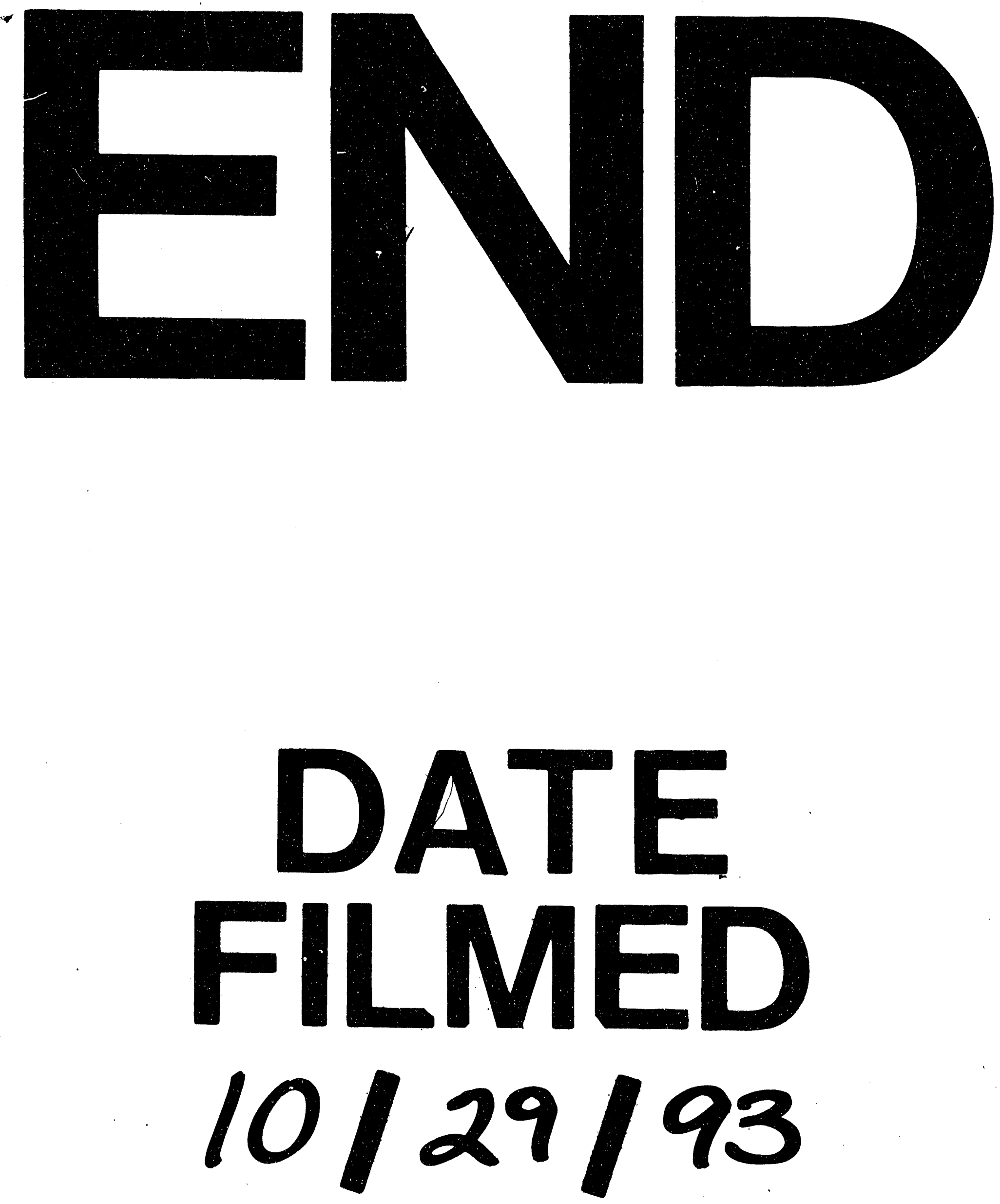
